Themenheft Nr. 40: CoViD-19 und die digitale Hochschulbildung. Irritationen, Einsichten und Programmatiken

Herausgegeben von Markus Deimann, Marios Karapanos und Klaus Rummler

\title{
Unterstützungsbedarfe Hochschullehrender in Lehramtsstudiengängen und Weiterbildungsangebote im (digitalen) Sommersemester 2020
}

\author{
Mariane Liebold ${ }^{1}$ (D) , Verena Odrig ${ }^{1}$, Juliane Tolle ${ }^{2}$ (D), Christine Dallmann ${ }^{1}$ (D) und Nadine \\ Schaarschmidt ${ }^{1}$ \\ ${ }^{1}$ Technische Universität Dresden \\ ${ }^{2}$ Technische Universität Chemnitz
}

\section{Zusammenfassung}

Die pandemiebedingten Veränderungen des Hochschulbetriebs im Sommersemester 2020 forderten Hochschuldozierende hinsichtlich ihrer digitalisierungsbezogenen Kompetenzen heraus. Das Vorwissen und die bis dato gesammelten Erfahrungen mit Blick auf eine digitale Lehre deckten sich oftmals nicht mit den neuen aus der Not heraus entstandenen Anforderungen an die Lehrenden. In den Lehramtsstudiengängen ist es unabdingbar eine medienpädagogische Handlungskompetenz an die Studierenden zu vermitteln, über die Dozierende im Hochschulzusammenhang selbst verfügen müssen. Doch welche Möglichkeiten wurden den Lehrenden zum Ausbau der eigenen Kompetenzen angeboten? Inwiefern wurden die während des Sommersemesters 2020 angebotenen Weiterbildungen den entsprechenden Bedarfen gerecht? Der vorliegende Beitrag stellt eine Studie vor, die sich mit den Weiterbildungsangeboten für Hochschullehrende an sächsischen Universitäten während des ‘digitalen, Sommersemesters befasst und diese thematisch mit den Unterstützungsbedarfen Dozierender in den Lehramtsstudiengängen der Technischen Universität Dresden vergleicht. Als Grundlage für diesen Vergleich dient ein vorab eigens entwickelter Kompetenzrahmen, welcher auf vorhandenen Konzepten und Modellen zu Medienkompetenz (Baacke 1997) und Medienpädagogischer Kompetenz (Blömeke 2000) beruht. 


\title{
Support Needs of University Lecturers in Teaching Degree Programs and Advanced
} Training Programs during the 'Digital' Summer Semester 2020

\begin{abstract}
The pandemic-related changes in university operations in the summer semester of 2020 challenged university lecturers with regard to their digitization-related competencies. The previous knowledge and the experience gained so far with regard to digital teaching often did not match the new requirements for teachers that arose out of necessity. In teacher training courses, it is essential to provide students with media pedagogical competence, which lecturers must have at their disposal in the university context. But what possibilities were offered to the teachers to develop their own competences? To what extent did the continuing education courses offered during the summer semester 2020 meet the corresponding needs? This study looks at the continuing education offerings for university lecturers at Saxon universities during the 'digital' summer semester and compares them thematically with the support needs of lecturers in the teaching degree programs at the Technische Universität Dresden. As a basis for this comparison, a competence framework is developed in advance and based on existing concepts and models of media competence (Baacke 1997), media pedagogical competence (Blömeke 2000).
\end{abstract}

1. Einführung - Auswirkungen der Pandemie auf den Lehrbetrieb an Hochschulen Die Folgen der CoViD-19-Pandemie haben die Hochschullehre weltweit geprägt. Die Universitäten und Hochschulen reagierten mit Schliessungen, um den neuen Abstandsregelungen gerecht werden zu können und damit eine Weiterverbreitung des neuartigen Virus zu unterbinden (vgl. Rashid und Yadav 2020, 340). Nur ein kleiner Teil der Hochschulen weltweit erlebte keinerlei Auswirkungen auf deren Lehrbetrieb. Dies waren überwiegend Hochschulen, die hauptsächlich Fernstudiengänge anbieten (vgl. Marinoni, Van't Land, und Jensen 2020, 23). Die übrigen Hochschulen stellten grösstenteils auf eine reine Online-Lehre um oder vorübergehend den Lehrbetrieb komplett ein (vgl. ebd.).

Im März 2020 verbreitete sich CoViD-19 auf dem europäischen Kontinent rasant (vgl. Crawford et al. 2020, 12). Die deutschen Hochschulen stellten die Präsenzlehre ein und gingen über zur Online-Lehre, wobei sich die Herangehensweisen der einzelnen Universitäten sowie bundeslandspezifisch unterschieden (vgl. ebd.). Der Wechsel zur vollständigen Online-Lehre konnte nicht langfristig vorbereitet werden, sondern erwuchs aus einer Not. Die daraus hervorgegangenen Angebote können in den meisten Fällen als «emergency eLearning» bezeichnet werden (vgl. Rashid und Yavad 2020, 341). Noch im März war den Entscheidungstragenden häufig unklar, wie das kommende Semester gestaltet werden könnte (vgl. Crawford et al. 2020, 12). 
Mangels Vorbereitungsmöglichkeiten kam es zu Defiziten im Zusammenhang mit dem Emergency eLearning. So «zeigte sich [...] zu Beginn der Pandemie ein massiver Nachholbedarf in diesem Bereich» (Graf et al. 2020).

Dem vorliegenden Beitrag liegt eine Studie zugrunde, die die Erfahrungen Lehrender in Lehramtsstudiengängen der TU Dresden im Umgang mit digitaler Lehre zu Beginn der Pandemie untersucht und sich davon ausgehend entsprechenden Weiterbildungsangeboten für Hochschullehrende widmet. Zunächst werden Forschungsstand, Forschungsmotivation Forschungsfrage sowie die methodische Herangehensweise dargelegt. Darauf folgt die Darlegung des im Rahmen des Projekts erarbeiteten Kompetenzrahmens (Schaarschmidt et al. 2020) und die anschliessende Vorstellung der Umfrageergebnisse bezüglich bestehender Kompetenzen aufseiten Hochschullehrender in Lehramtsstudiengängen an der TU Dresden, die auf einer Online-Befragung vom 12. Februar bis zum 14. April 2020 beruhen. ${ }^{1}$ Anhand der Ergebnisse wurden digitalisierungsbezogene Kompetenzen Lehrender in der Lehrpraxis sowie Unterstützungsbedarfe sichtbar. Daran schliesst sich die Vorstellung der Ergebnisse einer Recherche zu Weiterbildungsangeboten (vorrangig in Sachsen) an, die den Zeitraum April bis September 2020 in den Blick nimmt. Hier wurden Angebote, die digitalisierungsbezogene Kompetenzen Hochschullehrender adressieren oder zumindest Teilbereiche unterstützen, gesichtet und anhand des Kompetenzrahmens kategorisiert. Die Ergebnisse werden in einem letzten Schritt den Unterstützungsbedarfen der Hochschullehrenden gegenübergestellt. Darauf aufbauend werden Handlungsempfehlungen abgeleitet, die sich konkret auf Hochschuldozierende in Lehramtsstudiengängen beziehen.

\section{Forschungsstand, Forschungsmotivation und Forschungsfrage}

Die schnelle Umstellung von der Präsenzlehre hin zur Online-Lehre wirft zentrale Fragen der Implementierung der Online-Lehre auf und bietet damit ein spannendes Forschungsfeld (vgl. Krammer, Pflanzl, und Matischek-Jauk 2020). Die Frage nach den Bedarfen sowie den entsprechenden Weiterbildungsangeboten ist angesichts der aktuellen Bedeutung digitaler Lehre wichtiger denn je. Des Weiteren steht die Hochschullehre im Zentrum neuer Anforderungen, die an das Weiterbildungsangebot durch die Neuerungen in der Arbeitswelt gestellt werden. "Derartige Entwicklungsaufgaben sind eindeutig an die Hochschulen und dort insbesondere an die wissenschaftlichen Weiterbildungseinrichtungen gerichtet» (Bräutigam und Schindler 2021, 137). Damit wird die Untersuchung von Weiterbildungsangeboten zu einem zentralen Forschungsfeld im Sinne der gesamtgesellschaftlichen Innovationsfähigkeit.

1 Die Erhebung wurde in Kooperation mit der Professur für Didaktik der Informatik der TU Dresden durchgeführt. 
Toquero unterstreicht in diesem Zusammenhang die Bedeutung der Hochschullehre sowie der Untersuchung der Reaktionen und Veränderungen ihrer Organisationsweise während der Pandemie, um gesamtgesellschaftlich auf zukünftige Pandemien besser vorbereitet zu sein (vgl. Toquero 2020, 2).

Bereits vor den Veränderungen im Zuge der CoViD-19-Pandemie erforderte die Digitalisierung einen gleichzeitigen Shift auf Seiten der Hochschullehre. Anhand der Literatur in diesem Zusammenhang wird deutlich, welche Erfordernisse und Bestrebungen bereits vor Beginn der Pandemie für die Hochschulen von Interesse waren. So setzen sich etwa Matveeva, Akatova, Shcherbakov und Filinova (2020) in ihrer Untersuchung vor allem mit der Rolle der Digitalisierung und den daraus erwachsenden Anforderungen an die Hochschulbildung auseinander. Im Rahmen einer Umfrage machen sie Trends bei der Etablierung neuer Herangehensweisen an die Lehre aus und entwerfen aufbauend auf den von ihnen erhobenen Ergebnissen Handlungsempfehlungen für eine erfolgreiche Anpassung der Hochschullehre an die Herausforderungen der digitalen Transformation (ebd.).

Auch Ugurs (2020) Beitrag untersucht unabhängig von den Auswirkungen der Pandemie den Einsatz digitaler Lehrmittel sowie die Beweggründe, Vorgehensweisen und Erfahrungen beim Umgang damit seitens der Lehrenden und Lernenden in der Hochschulbildung und gibt einen Einblick in den Umgang und das Verständnis vor der Pandemie (vgl. Ugur 2020). Konkret mit den Erfordernissen der Digitalisierung und der Umsetzung in der Lehre im Rahmen des Lehramtsstudiums befasst sich der Beitrag von Lund und Agaard (2020).

Neben den Untersuchungen, welche sich mit den Anforderungen an Lehrkräfte im Zuge der Digitalisierung sowie ihren Hilfebedarfen auseinandersetzen, bilden Untersuchungen direkt zu den Auswirkungen der CoViD-19-Pandemie auf die Hochschullehre ein weiteres Feld. So vergleichen etwa Crawford et al. (2020) in ihrer Studie die Reaktionen der Hochschulen verschiedener Staaten. Auch die International Association of Universities befasst sich in ihrem Beitrag mit den Auswirkungen der CoViD-19-Pandemie auf die Hochschullehre weltweit (Marinoni, Van't Land, und Jensen 2020). Neben diesen Beiträgen, die sich vor allem mit der Lage der Hochschulen und ihrem Vorgehen auseinandersetzen, gibt es ausserdem Beiträge, die Handlungsempfehlungen für einen zukünftigen Umgang mit Krisensituationen und digitaler Lehre im Hochschulkontext formulieren (Rashid und Yadav 2020; Toquero 2020; Reinmann et al. 2020).

Ein weiteres Feld im Forschungsstand bilden Kompetenzrahmen und -modelle, welche sich mit den Anforderungen an Lehrkräfte befassen (Baacke 1997; Blömeke 2000; Holdener, Bellanger, und Mohr 2016; Sächsisches Staatsministerium für Kultus 2017; Tulodziecki 2012). Sie sind Grundlage für den hier verwendeten Kompetenzrahmen (Schaarschmidt et al. 2020). 
Der vorliegende Beitrag stellt vor diesem Hintergrund eine Studie vor, die sich den digitalisierungsbezogenen Kompetenzen der Hochschullehrenden widmet. Untersucht wurden Weiterbildungsmöglichkeiten, die den Lehrenden zum Ausbau ihrer digitalisierungsbezogenen Kompetenzen während des Sommersemesters 2020 in Sachsen angeboten wurden. Gleichzeitig soll durch den Abgleich zwischen den erhobenen Bedarfen hinsichtlich verschiedener Teilkompetenzen und den in Weiterbildungsangeboten adressierten Kompetenzanforderungen ermittelt werden, inwiefern die Angebote den Bedarfen gerecht werden und an welchen Stellen eventuell ein Ungleichgewicht festzustellen ist. Forschungsleitend sind demgemäss die folgenden Fragen: Welche Defizite bestehen hinsichtlich der digitalisierungsbezogenen Kompetenzen der Hochschullehrenden, um die Medienkompetenz von Schülerinnen und Schülern in der Schule zu fördern? Inwiefern können existierende Weiterbildungen im Hochschulkontext diese Defizite adressieren bzw. daran anknüpfen?

\section{Methodisches Vorgehen}

Die Untersuchung erfolgte anhand einer Fragebogen-Erhebung zu bestehenden Kompetenzen und Unterstützungsbedarfen Dozierender an der TU Dresden sowie einer Analyse von Weiterbildungsangeboten während des digitalen Semesters. Die Ergebnisse beider Teiluntersuchungen wurden sodann gegenübergestellt, verglichen und entsprechende Handlungsempfehlungen abgeleitet.

Im Rahmen einer Online-Befragung wurden Hochschullehrende in Lehramtsstudiengängen der TU Dresden vom 12. Februar bis zum 14. April 2020 befragt. Die Lehrenden wurden gebeten ihre Kompetenzen, Bedarfe sowie ihre aktuelle Lehrpraxis selbst einzuschätzen. Die Erhebung wurde mit Hilfe von LimeSurvey durchgeführt und umfasste überwiegend Multiple-Choice- sowie einige Freitext-Fragen. Die Auswertung der quantitativen Daten erfolgte deskriptiv. Die qualitativen Daten der Freitextaufgaben wurden mittels deduktiver Kategorienanwendung (vgl. Mayring 2015, 97) ausgewertet.

Zur Untersuchung der Weiterbildungsangebote wurden zunächst im Zeitraum vom März 2020 bis September 2020 Angebote gesammelt, welche die in einem zuvor entwickelten Kompetenzrahmen (vgl. Schaarschmidt et. al 2020) zusammengefassten Kompetenzen adressieren. Dabei wurden vorrangig Angebote (etablierter) sächsischer Weiterbildungsinstitutionen in den Blick genommen, die auf einschlägigen Portalen aufgeführt werden. ${ }^{2}$ Auf dieser Recherchearbeit aufbauend erfolgte eine

2 Als Beispiele sind u.a. Angebote des Hochschuldidaktischen Zentrums Sachsen (HDS), die Hochschuldidaktischen Weiterbildungsangebote an der TU Chemnitz, die Hochschule Meißen/Fortbildungszentrum (FoBiZ), das Zentrum für Weiterbildung der TU Dresden (ZfW), der Weiterbildungskatalog der TU Dresden und die Internetpräsenzen der Medienpädagogischen Zentren (SAEK) und der Lehramtskompass der Uni Leipzig, sowie überregional Angebote des Instituts für Medienpädagogik in Forschung und Praxis (JFF) zu nennen. 
inhaltliche Analyse der verschiedenen Angebote anhand der Kursbeschreibungen. Mittels deduktiver Kategorienanwendung wurde untersucht (vgl. Mayring 2015, 97), welche Kompetenzen in den Veranstaltungen ausgebaut oder vertieft werden sollen. Dabei wurden die Kursbeschreibungen anhand eines Kompetenzrahmens (siehe Kapitel «Digitalisierungsbezogene Kompetenzen - Kompetenzrahmen») klassifiziert und den entsprechenden Kompetenzbereichen zugeordnet, wobei einzelne Veranstaltungen mehrere Kompetenzbereiche adressierten.

Ausserdem wurde zwischen einer direkten und indirekten Adressierung unterschieden. Als indirekt adressiert wurden Kompetenzen im Kontext der entsprechenden Angebote eingestuft, wenn eine individuelle Transferleistung der Teilnehmenden für einen weiteren Ausbau erforderlich ist.

Die Analyse der Weiterbildungsangebote anhand der jeweils adressierten Kompetenzbereiche war Grundlage für die Gegenüberstellung mit den Ergebnissen aus der Erhebung zu bestehenden Kompetenzen und Unterstützungsbedarfen Lehrender in den Lehramtsstudiengängen der TU Dresden. Anhand der Gegenüberstellung konnten schliesslich Handlungsempfehlungen abgeleitet werden.

\section{Digitalisierungsbezogene Kompetenzen - zugrunde gelegter Kompetenzrahmen}

Die Medienkompetenz der Schülerinnen und Schüler in der Schule zu fördern, ist bereits seit Langem von grosser Bedeutung. Im Kontext der Digitalisierung ist dabei auch speziell von «digital literacies» die Rede (vgl. Lund und Aagaard 2020, 57). Neben der Bereitstellung und Zuverlässigkeit einer funktionierenden Infrastruktur sind dafür vor allem Kompetenzen aufseiten der Lehrenden zentral (vgl. Schaper 2020, 314; Blömeke 2000; Dallmann 2017). Angesichts dessen lassen sich Anforderungen an die Hochschullehre ableiten und das im besonderen Masse an die Lehrpraxis in Lehramtsstudiengängen (vgl. Tulodziecki 2012; 2017).

Um digitalisierungsbezogene Kompetenzen bei Hochschullehrenden zu erfassen, wurde im Rahmen des Projekts «DiKoLA - Digitalisierungsbezogene Kompetenzen im Lehramtsstudium» ausgehend von vorhandenen Konzepten und Modellen zu Medienkompetenz (Baacke 1997) und Medienpädagogischer Kompetenz (Blömeke 2000) ein Kompetenzrahmen entwickelt, der der Erhebung zugrunde gelegt werden konnte und bereits ausführlich dokumentiert ist (Schaarschmidt et. al 2020). Im Folgenden sei der Kompetenzrahmen mit Blick auf die Untersuchung zusammenfassend dargelegt.

Grundlegend ist eine Arbeitsdefinition digitalisierungsbezogener Kompetenzen in Anlehnung an die theoretische Grundlegung zu Medienkompetenz nach Baacke (1997, 98f). Medienkompetenz und damit auch digitalisierungsbezogene Kompetenzen adressieren alle Menschen und bilden damit auch für Lehrende die Grundlage. 
Lehrpersonen bzw. Lehramtsstudierende und Hochschuldozierende in lehramtsbezogenen Studiengängen sollten im Sinne medienpädagogischer und -didaktischer Zielsetzungen im Bildungskontext jedoch über weitere Kompetenzen verfügen, die über die Eigene Medienkompetenz hinausgehen und sich unter dem Terminus Medienpädagogischer Kompetenz, der die Eigene Medienkompetenz als Voraussetzung integriert, fassen lassen (vgl. Blömeke 2000; Tulodziecki 2012). Hinzukommen eher praxisorientierte Ansätze wie der Kompetenzrahmen der KMK (2016) in seiner Adaption durch das SMK (2017) oder das Digital Literacy Framework (Holdener, Bellanger, und Mohr, 2016) und Digital Competence Framework for Educators (DigCompEdu) (2017).

In Anlehnung an die Medienpädagogische Kompetenz nach Blömeke (2000) wurden für den hier entwickelten Kompetenzrahmen neben der Eigenen Medienkompetenz weitere Teilbereiche adaptiert und folgendermassen in den vorliegenden Kompetenzrahmen (vgl. Abbildung 1) integriert:

1. Mediendidaktische Kompetenz $z^{3}$

2. Medienerzieherische Kompeten $z^{4}$

3. Sozialisationsbezogene Kompetenz im Medienzusammenhang ${ }^{5}$

4. Hochschulentwicklungskompetenz im Zusammenhang mit digitalen Medien ${ }^{6}$

3 Mediendidaktische Kompetenz umfasst «die Fähigkeit zur reflektierten Verwendung von Medien und Informationstechnologien in geeigneten Lehr- und Lernformen und deren Weiterentwicklung» (Blömeke 2000, 157). Als Beispiel kann der Einsatz von Medien und Informationstechnologien in der Lehre gesehen werden, aber auch die Gestaltung digitaler Lehr- und Lernformen.

4 Die Medienerzieherische Kompetenz umfasst die «Fähigkeit, Medienthemen im Sinn pädagogischer Leitideen im Unterricht behandeln zu können» (ebd., 159) und betrifft damit die Realisierung der Erziehungsaufgaben im Bereich der Medien und Informationstechnologien.

5 Die Sozialisationsbezogene Kompetenz im Medienzusammenhang wird als «Fähigkeit zur konstruktiven Berücksichtigung der Lernvoraussetzungen beim medienpädagogischen Handeln» (ebd., 162) definiert. Hierzu zählen bspw. das Erfassen der Lebenswelten von Lernenden, das Verstehen ihres Mediennutzungsverhalten.

6 Die Hochschulentwicklungskompetenz im Medienzusammenhang entspricht der Schulentwicklungskompetenz bei Blömeke (ebd.) als weiterer Bestandteil Medienpädagogischer Kompetenz: die «Fähigkeit zur innovativen Gestaltung der Rahmenbedingungen medienpädagogischen Handelns» (ebd., 165). Darunter fallen hier entsprechend die Wahrnehmung der Bedeutung von Medien für die Professionalität des Lehrberufs und für die Hochschulentwicklung sowie die Gestaltung der gesellschaftlichen und schulischen Bedingungen von Medienpädagogik in der Hochschule. 
Zusätzlich wurde der vorliegende Kompetenzrahmen durch folgende Kategorien ergänzt:

5. wissenschaftlichen Kompetenz in Bezug auf digitale Medien ${ }^{7}$ (in Anlehnung an Holdener, Bellanger, und Mohr 2016),

6. Professionalisierung beruflicher Kommunikations- und Kollaborationskompetenzen $^{8}$ (vgl. DigCompEdu, 2017; Holdener, Bellanger, und Mohr 2016).

\section{Monitor - Digitalisierungsbezogene Kompetenzen Hochschullehrender der TU \\ Dresden zu Beginn der CoViD-19-Pandemie}

Anhand des Kompetenzrahmens wurde eine Erhebung zu den verschiedenen Kompetenzbereichen bei Hochschulehrenden in den Lehramtsstudiengängen der TU Dresden vorgenommen. Der daraus hervorgegangene Monitor gibt Einblick in den Erfahrungsstand Hochschuldozierender direkt vor den Auswirkungen der Pandemie. Für den Monitor wurden im Zeitraum vom 12. Februar bis zum 14. April 2020 Lehrende per Selbsteinschätzung online befragt. Die Erarbeitung der Fragen erfolgte in Zusammenarbeit der Professur für Medienpädagogik und der Professur für Bildungstechnologie sowie der Professur für Didaktik der Informatik der TU Dresden.

Die Erhebung adressierte Hochschuldozierende der TU Dresden, die Lehrveranstaltungen für Lehramtsstudierende anbieten. Teilgenommen haben 89 Personen, allerdings wurden in einigen Fällen nicht alle Fragen beantwortet. Die Beteiligung war hoch, jedoch ist die Stichprobe nicht repräsentativ. Die Anzahl der möglichen Zielgruppenpersonen konnte nicht ermittelt werden, da der Lehramtsbereich an der TU Dresden interdisziplinär arbeitet. Die Erhebung wurde mit Hilfe von LimeSurvey durchgeführt und umfasste überwiegend Multiple-Choice- sowie einige Freitext-Fragen.

7 Unter wissenschaftlicher Kompetenz in Bezug auf digitale Medien versteht man die «Nutzung und Erzeugung von digitalen Daten, Quellen, Beweisen, Untersuchungsmethoden, Publikationen, um wissenschaftliche Ziele zu erreichen.» (Holdener, Bellanger, und Mohr 2016, 71). Hinzu kommen die Integration digitaler bzw. digitalisierungsbezogener wissenschaftlicher Ergebnisse in die Lehre zur Verbesserung der Lehre sowie die Nutzung digitalisierungsbezogener wissenschaftlicher Ergebnisse zur Bewertung neuer Trends.

8 Der Teilbereich der Professionalisierung beruflicher Kommunikations- und Kollaborationskompetenzen setzt sich aus den Kategorien Kommunikation und Kollaboration von Holdener, Bellanger, und Mohr (2016) und berufliches Engagement des DigCompEdu (European Commission 2017) zusammen. Neben der Fähigkeit zur Nutzung digitaler Kommunikationsmittel und der aktiven Teilhabe in sozialen Netzwerken für Lernen, Lehren und Forschen (Holdener, Bellanger, und Mohr 2016, 71) werden die Fähigkeit zum Austausch mit Kolleginnen und Kollegen sowie die organisatorische Kommunikation mit Lernenden und Dritten (vgl. Kategorie des beruflichen Engagements des DigCompEdu, European Commission 2017) betont. Weiterhin fokussiert dieser Teilbereich nicht nur die Kommunikation und Kollaboration, sondern auch die Fähigkeit, digitale Technologien zur Verbesserung der Lehre und für die eigene berufliche Weiterbildung zu nutzen bzw. einzusetzen (European Commission 2017). 
Im Wesentlichen nahmen wissenschaftliche Mitarbeitende an der Umfrage teil, welche verschiedenen Fachbereichen angehören. ${ }^{9}$ Unter den Teilnehmenden sind alle Altersgruppen vertreten. ${ }^{10}$ Auffällig ist, dass sich die meisten der Befragten, gemessen an der Zuordnung der Mehrzahl ihrer abgehaltenen Lehrveranstaltungen, mit $49 \%$ in den Fachwissenschaften verorten, $28 \%$ in der Fachdidaktik, in den Bildungswissenschaften $17 \%$ und $5 \%$ gaben keine Antwort.

In einer ersten Frage wurden die Aspekte des Kompetenzrahmens «Kompetenzen in der digitalen Welt» der KMK (2017) in der Fassung der SMK-Konzeption «Medienbildung und Digitalisierung» (2017) in abgewandelter Form hinsichtlich der Thematisierung in Lehrveranstaltungen abgefragt. Gleicht man die Selbsteinschätzungen mit den Vorgaben der KMK (2017) ab, so ergibt sich, dass die Bereiche Präsentieren, Kommunizieren und Kooperieren sowie Suchen und Verarbeiten am häufigsten thematisiert werden. Die Bereiche Produzieren, Problemlösen und Handeln sowie Schützen und Sicher Agieren werden hingegen gar nicht bis selten behandelt (siehe Abbildung 1).



Abb. 1.: Häufig thematisierte Aspekte.

9 Den höchsten Anteil bildet der Bereich Geistes- und Sozialwissenschaften mit $57 \%$ der Befragten, gefolgt vom Bereich Mathematik und Naturwissenschaften mit $26 \%(n=81)$.

10 Die grösste Gruppe findet sich unter den Personen zwischen 31 und 40 Jahren (32 \%). 
Im Anschluss daran wurde die Art und Weise der Thematisierung dieser Felder in der Lehrveranstaltung erfragt. Hier konnten die Teilnehmenden in einem Freitextfeld antworten. Anhand ihrer Antworten wurden die folgenden fünf Kategorien ${ }^{11}$ abgeleitet:

1. Thematisieren erfolgt im Kontext anderer vordergründiger Themen (26 Zuordnungen)

2. Hinweise/Tipps (17 Zuordnungen)

3. Praxis/Selbsterfahrung (14 Zuordnungen)

4. Reflexion (7 Nennungen)

5. Vorbild-Funktion (6 Nennungen)

Nach den von ihnen verwendeten Technologien und Geräten befragt, gaben die Teilnehmenden mehrheitlich an, dass sie vorrangig Laptop/Tablet, Beamer, Kreidetafel/Whiteboard, Interaktive Tafel und Overheadprojektor in ihren Lehrveranstaltungen einsetzen..$^{12} \mathrm{Zu}$ den häufig genutzten Softwareanwendungen zählten laut der Umfrage die Dokumentenbereitstellung, die Nutzung von Lernplattformen (wie z. B. OPAL), Präsentationssoftware, Software zum Austausch sowie Filme oder Video-Software (siehe Tabelle 1).

\begin{tabular}{|lcccc|}
\hline n=89 & $\begin{array}{c}\text { wöchent- } \\
\text { lich/monat- } \\
\text { lich }\end{array}$ & $\begin{array}{c}\text { selten/nie } \\
\text { kenne ich } \\
\text { nicht }\end{array}$ & $\begin{array}{c}\text { keine Ant- } \\
\text { wort }\end{array}$ \\
\hline $\begin{array}{l}\text { Kooperationssoftware (z. B. Etherpad, } \\
\text { Wiki) }\end{array}$ & $20 \%$ & $64 \%$ & $3 \%$ & $12 \%$ \\
Software zum Austausch (z. B. Forum) & $45 \%$ & $43 \%$ & $1 \%$ & $11 \%$ \\
Medienplattform (z. B. YouTube) & $41 \%$ & $48 \%$ & $0 \%$ & $11 \%$ \\
Dokumentenbereitstellung (z. B. pdf) & $88 \%$ & $2 \%$ & $0 \%$ & $11 \%$ \\
Lernplattform (z. B. Opal) & $85 \%$ & $5 \%$ & $0 \%$ & $10 \%$ \\
Präsentationssoftware (z. B. Power- & $84 \%$ & $5 \%$ & $0 \%$ & $11 \%$ \\
Point) & $46 \%$ & $39 \%$ & $1 \%$ & $12 \%$ \\
Filme oder Video-Software & $8 \%$ & $78 \%$ & $0 \%$ & $15 \%$ \\
Podcast & $7 \%$ & $77 \%$ & $4 \%$ & $12 \%$ \\
Simulation & $2 \%$ & $48 \%$ & $35 \%$ & $15 \%$ \\
Screencast-Software (z. B. Camtasia) & $12 \%$ & $57 \%$ & $16 \%$ & $15 \%$ \\
Software zur Erstellung von Tests/Auf- & & & & \\
gaben (z. B. Onyx) & $14 \%$ & $43 \%$ & $29 \%$ & $13 \%$ \\
Audience-Response-System (z. B. & & & & \\
AMCS) & & & & \\
\hline
\end{tabular}

Tab. 1.: Nutzung von Softwareanwendungen.

11 Die Kategorienbildung erfolgte in Form einer zusammenfassenden Analyse.

12 Hingegen nie genutzte Technologien sind VR-System/-Brille, 3D-Drucker, Multitouch-Display, Mikrocontroller/Einplatinencomputer und Clicker-System. Zu den am wenigsten bekannten Technologien zählen die Interaktive Tafel/Active Board und der 3D-Drucker. 
Auf die Frage nach dem Einsatz von Lernszenarien gaben die Teilnehmenden am häufigsten an, das Szenario «Flipped Classroom» (15\%) einzusetzen, gefolgt von "Blended Learning»-Szenarien (14\%) und E-Portfolio-Systemen (12\%). Auffällig sind die Ergebnisse der Zuordnung «kenne ich nicht», wo das E-Assessment und auch das E-Portfolio-System am häufigsten genannt werden.

Im Zuge der Umfrage wurde auch die Reaktion der Lehrenden auf neue Anforderungen durch die Digitalisierung erfragt. Bei der Auswertung der Freitextfeldantworten wurden folgende Kategorien ermittelt:

6. Methodisch-didaktische Anpassung ${ }^{13}$ (40 Zuordnungen)

7. Inhaltliche Anpassung ${ }^{14}$ (13 Zuordnungen)

8. Organisation ${ }^{15}$ (11 Zuordnungen)

9. Keine Änderung (6 Zuordnungen)

Im weiteren Verlauf wurden Angaben zum Kontaktieren von Servicestellen oder Ansprechpartnerinnen und -partnern erhoben. Am meisten kontaktiert wurden Kolleginnen und Kollegen mit $64 \%$, gefolgt vom Zentrum für Informationsdienste und Hochleistungsrechnen der TU Dresden (ZIH) mit $45 \%$ und Online-Informations- und Weiterbildungsangeboten mit $44 \%{ }^{16}$. Ein Grossteil der Befragten weiss demnach, wohin er sich themenbezogen wenden können und nutzen demzufolge das $\mathrm{ZIH}$, den E-Learning-Support der TU Dresden oder auch das Zentrum für Weiterbildung (ZfW, siehe Tabelle 2).

\begin{tabular}{|ll|}
\hline $\mathrm{n}=86$ & \\
\hline Kolleginnen und Kollegen, die sich damit auskennen & $64 \%$ \\
Zentrum für Informationsdienste und Hochleistungsrechnen (ZIH) & $45 \%$ \\
Informations-/Weiterbildungsangebote online & $44 \%$ \\
E-Learning-Support der TU Dresden & $40 \%$ \\
Zentrum für Weiterbildung (ZfW) & $24 \%$ \\
externe Weiterbildungen & $22 \%$ \\
E-Learning-Beauftragte der Fakultät & $13 \%$ \\
Hochschuldidaktisches Zentrum Sachsen (HDS) & $12 \%$ \\
Zentrum für interdisziplinäres Lernen und Lehren (ZiLL) & $12 \%$ \\
\hline Koordinierungsstelle «Digitalisierung in der Lehrerbildung» & $6 \%$ \\
\hline
\end{tabular}

Tab. 2.: Kontaktierte Servicestellen sowie Ansprechpartnerinnen und -partner.

13 Z. B. Nutzung von Tools oder Reflexion medialer Werkzeuge und Inhalte.

14 Z. B. Medien als Inhalt selbst oder Erweiterung der Inhalte.

15 Z. B. Vor- und Nachbereitung oder zunehmende Komplexität.

16 Hier waren Mehrfachnennungen möglich. 
Auf die Frage nach ihren Unterstützungsbedarfen, um digitalisierungsbezogene Kompetenzen in ihren Lehrveranstaltungen stärker thematisieren bzw. fördern zu können, wünschen sich die Teilnehmenden am häufigsten Weiterbildungen für Lehrende $(51 \%) .{ }^{17}$

Daran anknüpfend wurden gewünschte Fortbildungsthemen erfragt. An erster Stelle wünschen sich die Befragten mit 28 Nennungen Themen hinsichtlich digitaler Tools und Werkzeuge ${ }^{18}$. An zweiter Stelle werden digitale Lehr-Lern-Szenarien aufgeführt mit 14 Nennungen. Themen über digitale Medien wünschen sich sieben Personen, darunter fallen die Themen Datenschutz, Inklusion und Rechtsfragen. Fünf Personen gaben bei sonstigen Themenwünschen bzw. fachbezogenen Wünschen die Schulrelevanz von Mediennutzung und neue Lernkulturen an.

Des Weiteren wurden erwünschte Fortbildungsthemen für die eigene Fortentwicklung bezüglich der Vermittlung digitalisierungsbezogener Kompetenzen in den jeweiligen Lehrveranstaltungen erfragt. Genannt werden überwiegend Themen über digitale Medien, wie beispielsweise Urheberrecht, Datenschutz oder Medienkritik. Mediendidaktik folgt an zweiter Stelle mit Inhalten wie beispielsweise Modellen zur Vermittlung oder Kompetenzrastern. Folgend werden ausserdem digitale Tools für Schülerinnen und Schüler bzw. Lernende genannt.

Es folgte die Frage nach den Formaten, welche sich Lehrende bei Fortbildungen wünschen. Zu den meistgenannten zählen hier Präsenz-Formate mit $43 \%$. Es folgen Online-Fortbildungen mit $37 \%$ und Blended Learning mit $33 \%$.

Von 89 Teilnehmenden gaben lediglich $10 \%$ an, bereits ein Good-Practice Beispiel in ihrer Lehrveranstaltung einzusetzen, um digitalisierungsbezogene Kompetenzen zu adressieren. Unter den genannten Beispielen finden sich überdies schwerpunktmässig didaktische Umsetzungen, medienpädagogische Inhalte werden vernachlässigt.

Anhand des Monitors lassen sich Unterstützungsbedarfe der Lehrenden ableiten, die im Anschluss an eine Betrachtung der besonderen Herausforderungen des «digitalen> Sommersemesters und die Vorstellung der Ergebnisse der Untersuchung der Weiterbildungsangebote im Rahmen von Handlungsempfehlungen eingängig und in Kontext gesetzt, thematisiert werden.

17 Im Anschluss daran folgen die Wünsche nach mehr Personal (45 \%), finanzielle Mittel für Anschaffungen, wie z. B. Geräte (40 \%) oder technischen Support (36\%), Austausch mit Kolleginnen und Kollegen, z. B. durch Arbeitskreise (35\%), Beratungen durch Expertinnen und Experten, z. B. durch Servicestellen (31\%), finanzielle Mittel für (Lehr)projekte (23\%) oder Sonstiges (häufigste Nennung: «Zeit») mit $15 \%$.

18 Hierunter wurden u. a. White Board, Audience Response Systeme oder der Überblick über verschiedene Werkzeuge genannt. 


\section{Herausforderungen und Reaktionen zu Beginn des (digitalen) Semesters}

Die Anforderungen an den Kenntnisstand der Lehrenden mit Blick auf deren digitalisierungsbezogene Kompetenzen haben sich durch die Pandemie schlagartig weiter erhöht. Die Digitalisierung wurde beschleunigt. Graf et al. zufolge übernahmen die Universitäten zunächst die Aufgaben der Beschaffung von Lizenzen sowie die Bereitstellung allgemeiner Informationen zur digitalen Lehre (vgl. Graf et al. 2020). Die Fakultäten spielten laut der Autorin und den Autoren vor allem bei der personellen Unterstützung während der Umstellung eine Rolle (vgl. ebd.).

Die Umstellung auf nahezu reine Online-Lehre setzte neue Ansätze der pädagogischen Arbeit voraus und wurde damit zu einer Herausforderung für die Fakultäten, da die meisten relativ unvorbereitet diese Umstellung vollzogen. Marinoni et al. (2020) kommen dennoch zu dem Schluss, dass die verschiedenen Formen des Emergency eLearnings für die Lernprozesse an den Hochschulen gewinnbringender als ein völliger Ausfall der Lehrveranstaltungen waren.

In der von Graf et al. (2020) durchgeführten Studie zeigt sich eine klare Unterscheidung zwischen langfristig geplanter digitaler Lehre und «Emergency Remote Teaching». ${ }^{19}$ Neben der Planung und Vorbereitung verwiesen die von ihnen befragten Studienteilnehmenden ebenfalls auf die Notwendigkeit einer gleichzeitigen Veränderung der didaktischen Herangehensweise. Besondere Defizite infolge der Veränderungen machen sie vorrangig in den Bereichen «Forschendes Lehren» und bezüglich «intensive[r] Diskussionen» aus, «zumindest wenn das Lehrpersonal in digitaler Didaktik nicht (extensiv) geschult ist» (ebd.).

Anhand der Studie von Graf et al. wird deutlich, dass der Stand der digitalen Lehre vor allem mit Blick auf die digitalisierungsbezogenen Kompetenzen des Lehrpersonals zu Beginn der Pandemie nur unzureichend ausgebaut war (ebd.). Reinmann et al. (2020) stellen angesichts ihrer Studie zu den Erfahrungen Hochschuldozierender vor dem «digitalen) Semester ebenfalls fest, dass nur ein geringer Prozentsatz der Lehrenden über Erfahrungen in der reinen Online-Lehre verfügte (6\%). Gerade einmal $4 \%$ sammelten vor der Pandemie Erfahrungen zu E-Assessments und OnlineTests (ebd.).

19 Darunter verstehen die Autorin und die Autoren den schnellen Wechsel hin zur Online-Lehre in Reaktion auf die Pandemie. 


\section{Weiterbildungsangebote im ‘digitalen) Sommersemester 2020}

Weiterbildungen als feste Bestandteile lebenslangen Lernens nehmen in einer von Veränderungen gezeichneten Gesellschaft einen entscheidenden Stellenwert ein (Bräutigam und Schindler 2021, 137). Sie dienen als Instrument, um der mit der Digitalisierung einhergehenden «hohe[n] Dynamik der Veränderung nachgefragter Kompetenzen» gerecht zu werden (Duchêne et al. 2020, 10). Im Zuge der veränderten Situation unter Pandemiebedingungen ist das Unterstützungsbedürfnis der Lehrenden zur Bewältigung ihrer Aufgaben innerhalb dieses für die meist neuen Settings gestiegen, während die entsprechenden Unterstützungsangebote nur nachträglich ergänzt und weiter ausgebaut werden konnten (Reinmann et al. 2020, 2).

Basierend auf der Erhebung unter Dozierenden in der Lehramtsausbildung im Frühjahr konnten bereits Defizite festgestellt werden, die die Befragten per Selbsteinschätzung mit Blick auf ihre digitalisierungsbezogenen Kompetenzen ausmachen.

Als häufigste Aspekte für Weiterbildungsbedarfe wurden Präsentieren und Kommunizieren eingeschätzt, was auch auf obligatorische Prüfungsleistungen oder die allgemeine Organisation einer Lehrveranstaltung zurückgeführt werden könnte und nicht auf das Ziel einer kompetenten zukünftigen Lehrpersonenschaft. Auch bei Fragen zu in Lehrveranstaltungen verwendeten Technologien/Geräten sowie Softwareanwendungen nennen Lehrende eher (altbewährtes): PowerPoint, die Lernplattform OPAL etc. Dies lässt sich indirekt mit der Frage, an wen sich Lehrende bei digitalisierungsbezogenen Anliegen wenden, kombinieren: An erster Stelle stehen dabei erfahrenere Personen aus dem Kollegium, was eine zügige Integration neuerer Anwendungen bremsen kann. Der Grossteil kennt allerdings auch die Informationsund Weiterbildungsangebote, die wahrgenommen werden könnten, oder zumindest die jeweils zuständigen Stellen, die bei der Integration Unterstützung anbieten. ${ }^{20}$

Abzuleitende Defizite der Lehrenden in der Lehramtsausbildung der TU Dresden, bezogen auf die Kompetenzbereiche des Kompetenzrahmens (siehe Kapitel 4) sind u. a. die Eigene Medienkompetenz (MK), die Medienerzieherische Kompetenz (MeK), die Wissenschaftliche Kompetenz (WK) in Bezug auf digitale Medien und die Professionalisierung beruflicher Kommunikations- und Kollaborationskompetenzen (PKK). Die Umfrage ist zwar nicht repräsentativ und wirft nur punktuelle Schlaglichter auf die Kompetenzbereiche, erlaubt jedoch einen Einblick in den Ist-Zustand unter den Lehrenden in der Lehramtsausbildung zu Beginn der Pandemie. So kann angesichts der Ergebnisse keine akut prekäre Situation mit Blick auf die digitalisierungsbezogenen Kompetenzen festgestellt werden, jedoch wird deutlich, dass digitalisierungsbezogene Aspekte selten direkt in der Lehre adressiert werden, ausser sie sind vermeintlich zielführend mit Blick auf eine Prüfungsleistung oder eine vereinfachte Organisation einer Lehrveranstaltung.

20 Gründe dafür, dass entsprechende Angebote (des ZIH, E-Learning-Support, ZfW, Zentrum für interdisziplinäres Lernen und Lehren (ZiLL) ...) eher selten genutzt werden, wurden in der Umfrage zunächst nicht erhoben. 
Neben der Befragung Lehrender wurden im Rahmen der vorliegenden Untersuchung Weiterbildungsangebote recherchiert, welche digitalisierungsbezogene Kompetenzen (siehe Tabelle 3) Hochschullehrender adressieren oder zumindest Teilbereiche ansprechen. ${ }^{21}$ Die Recherche bezieht sich vorrangig auf das Bundesland Sachsen. Die ermittelten Angebote sind tabellarisch aufgeführt und nach den verschiedenen Institutionen unterteilt. Jeder Veranstaltung wurden jeweils Kompetenzbereiche anhand ihrer Kursbeschreibung und mittels deduktiver Kategorienanwendung zugeordnet (vgl. Mayring 2015, 97). Gleichzeitig gilt zu unterscheiden, ob die verschiedenen Kompetenzbereiche direkt adressiert werden oder ob es einer individuellen Transferleistung der Teilnehmenden bedarf, um bestimmte Kompetenzbereiche im Zuge der Weiterbildungen auszubilden. ${ }^{22}$

21 Eigene Medienkompetenz (MK), Mediendidaktische Kompetenz (MdK), Medienerzieherische Kompetenz (MeK), Sozialisationsbezogene Kompetenz im Medienzusammenhang (SbK), Hochschulentwicklungskompetenz im Zusammenhang mit digitalen Medien (HSeK), Wissenschaftliche Kompetenz in Bezug auf digitale Medien (WK), Professionalisierung beruflicher Kommunikations- und Kollaborationskompetenzen (PKK).

22 Bei der Erhebung der Weiterbildungsangebote wurden folgende Programme und Institutionen berücksichtigt: Clearingstelle Medienkompetenz der Deutschen Bischofskonferenz an der KH Mainz, E-Teaching Zertifikat für Hochschullehrende (OPAL/Zertifikatskurs 2020), Freie Selbstkontrolle Fernsehen, Hochschuldidaktische Weiterbildungsangebote an der TUC, Hochschuldidaktisches Zentrum Sachsen, Hochschule Koblenz - Institut für Forschung und Weiterbildung (IfW), Hochschule Meißen/Fortbildungszentrum (FoBiZ), Institut für Medienpädagogik in Forschung und Praxis (JFF), Lehrpraxis im Transfer+/ LiT.Shortcuts (Universität Leipzig, Dresden, Chemnitz) SAEK, Universität Leipzig, Zentrum für Weiterbildung Dresden (ZfW). 


\begin{tabular}{|c|c|c|c|c|c|c|c|c|}
\hline $\begin{array}{l}\text { Veran- } \\
\text { stalter }\end{array}$ & Workshops & MK & MdK & MeK & SbK & HSeK & WK & PKK \\
\hline \multirow[t]{4}{*}{ HDS } & Weblogs als alternatives Prüfformat & id & $d$ & & & & & id \\
\hline & Diagnostisches Assessment & d & $d$ & & & & id & id \\
\hline & Formatives Assessment & $d$ & $d$ & & & & id & id \\
\hline & Summatives Assessment & $d$ & d & & & & id & id \\
\hline IfW & Digitalisierung in den Handlungsfeldern der Kinder- und Jugendarbeit & d & & d & d & & id & \\
\hline \multirow[t]{7}{*}{ ZfW } & $\begin{array}{l}\text { Digitalen Wandel in der sächsischen Hochschulbildung gemeinsam } \\
\text { gestalten }\end{array}$ & & & & & $d$ & id & $d$ \\
\hline & $\begin{array}{l}\text { Programm für Lehrende: Vom Spiel lernen - Motivation und Transfer } \\
\text { durch Gamifizierung der Lehre }\end{array}$ & & d & id & & & & \\
\hline & $\begin{array}{l}\text { Intellectual Property Rights II - Urheber-, Medien- und Internetrechts } \\
\text { für Nichtjuristen (Zertifikatskurs) }\end{array}$ & $d$ & & & & $d$ & & \\
\hline & Programm für Lehrende: Online-Lehre an Hochschulen & & $d$ & & & & & $d$ \\
\hline & OER-Einsatz in der Lehre & $d$ & d & & & $d$ & & id \\
\hline & $\begin{array}{l}\text { Programm für Lehrende: Digital Workspace meets LEHRWERKSTATT ... } \\
\text { die digitale Transformation }\end{array}$ & & d & & & & & d \\
\hline & $\begin{array}{l}\text { Programm für Lehrende: Tiefenlernen fördern - Selbststudium und } \\
\text { Online- bzw. Präsenzlehre aufeinander abstimmen }\end{array}$ & & d & & & & & \\
\hline \multirow[t]{2}{*}{ TUC } & $\begin{array}{l}\text { Online-Workshop: Lehre digital gestalten - E-Learning in der Hoch- } \\
\text { schullehre }\end{array}$ & d & $d$ & & id & & & \\
\hline & $\begin{array}{l}\text { Digital Workspace: digital. Analog oder beides? Passende Konzepte für } \\
\text { die eigene Lehre entwickeln I digital }\end{array}$ & & $d$ & & & & & \\
\hline \multirow[t]{8}{*}{ SAEK } & Grundlagen des Visual Thinking (Online-Seminar) & & $d$ & $d$ & & & & \\
\hline & Lehren und Lernen: digital und online - (Online-Seminar) & & $d$ & d & & & & id \\
\hline & $\begin{array}{l}\text { Medienpädagogische Projekte praktisch umsetzen - SAEK Internet Days } \\
\text { (Online-Seminar) }\end{array}$ & $d$ & d & d & id & & & \\
\hline & SAEK Internet Days: Sicher im Netz (Online-Seminar) & d & & & & & id & \\
\hline & $\begin{array}{l}\text { Praxis Lehrfilme produzieren (mit PowerPoint, Webcam, Smartphone } \\
\text { oder Tablet) (Online-Seminar) }\end{array}$ & d & $d$ & & & & & \\
\hline & Urheberrecht im (digitalen) Unterricht (Online-Seminar) & d & d & & & & & \\
\hline & $\begin{array}{l}\text { Die Welt des Hörens - Alles rund um die Podcast- und Hörspielproduk- } \\
\text { tion }\end{array}$ & d & $d$ & & & & & \\
\hline & Big Data - Was unsere Daten über uns verraten (Online-Seminar) & d & & & & & id & \\
\hline FoBiZ & $\begin{array}{l}\text { Schulungsangebote für Dozentinnen und Dozenten, u. a. «Videotutori- } \\
\text { als/Lernvideos erstellen» }\end{array}$ & $d$ & d & & & & & \\
\hline $\begin{array}{l}\text { Clea- } \\
\text { ring- } \\
\text { stelle } \\
\text { MK }\end{array}$ & Zertifikatskurs medienpädagogische Praxis (mepps) & d & d & d & d & & id & id \\
\hline fsf & Grundlagenseminar Jugendmedienschutz & d & & d & & & & \\
\hline \multirow[t]{3}{*}{ JFF } & Medien_Weiter_Bildung \#teilhabe & d & d & d & d & & & \\
\hline & Medien_Weiter_Bildung \#gender & d & d & d & d & & & \\
\hline & Medien_Weiter_Bildung Rechtliche Grundlagen & d & & & & & d & \\
\hline \multirow{9}{*}{$\begin{array}{l}\text { LiT. } \\
\text { Short- } \\
\text { cuts }\end{array}$} & $\begin{array}{l}\text { Was sind digitale Kompetenzen und wie kann man sie bei Studierenden } \\
\text { fördern? }\end{array}$ & d & d & $d$ & & $d$ & & \\
\hline & Lehren und Lernen mit Online-Videos, Erklärfilmen und Audiobeiträgen & d & d & & & d & & \\
\hline & $\begin{array}{l}\text { Förderung der studentischen Beteiligung und Interaktion in der digita- } \\
\text { len Lehre }\end{array}$ & & d & & & & & \\
\hline & Einsatz von Weblogs in der Hochschullehre & $d$ & d & & & & & id \\
\hline & Asynchrone Lehre auf Moodle gestalten & d & d & & & & & \\
\hline & Urheberrechtliche Rahmenbedingungen der Hochschullehre & d & d & & & & & \\
\hline & Studentische Programmier- und Digitalkenntnisse fördern & d & d & d & & & & \\
\hline & $\begin{array}{l}\text { Open Educational Resources (OER) in der digitalen Hochschullehre: } \\
\text { Prinzipien - Rechtliche Grundlagen - Umsetzung }\end{array}$ & d & d & & & & d & \\
\hline & E-Teaching Zertifikat für Hochschullehrende & id & $\mathrm{d}$ & & & & & \\
\hline
\end{tabular}

Tab. 3.: Weiterbildungsangebote, Kompetenzbereiche ( $d=$ direkt adressiert, id = indirekt adressiert). 
Die Recherche zu Weiterbildungsangeboten, die die Kompetenzbereiche des eingangs vorgestellten Kompetenzrahmens fördern bzw. Defizite diesbezüglich direkt adressieren, war sehr ertragreich. So konnten im Zeitraum zwischen März 2020 und August 2020 verschiedene Angebote zu Weiterbildungen, welche sich direkt oder indirekt an Hochschuldozierende in den Lehramtsstudiengängen richten, an Standorten wie Dresden, Chemnitz, Zittau/Görlitz und Leipzig ausgemacht werden. ${ }^{23}$ Die Veranstaltungen unterscheiden sich hinsichtlich der Formate (Online, Präsenz). Hierbei sind jedoch die gegebenen Bedingungen angesichts des (digitalen〉 Semesters sowie die Grenzen der dargelegten Studie zu beachten. Da lediglich die in den Kursbeschreibungen adressierten Kompetenzbereiche untersucht wurden, erfolgte hier keinerlei Qualitätskontrolle der tatsächlichen Veranstaltungen. Die Studie kann demnach keine Aussagen dazu treffen, wie erfolgreich die verschiedenen Angebote umgesetzt wurden und welche Formate möglicherweise geeigneter sind. Neben den Formaten variiert der zeitliche Rahmen der recherchierten Angebote. Zum einen gibt es Tagesworkshops, andere Angebote erstrecken sich hingegen über mehrere Wochen oder gar Monate. Ebenso unterscheiden sich die Angebote in Hinblick auf die Kosten. So werden beispielsweise viele kostenfreie Angebote für Angestellte sächsischer Hochschulen bereitgestellt. Nur wenige Weiterbildungsangebote sind preisintensiv. Dementsprechend sind die Weiterbildungen zum Ausbau digitalisierungsbezogener Kompetenzen für die Lehramtsausbildung nicht zwangsläufig zeitaufwendig bzw. mit hohen Kosten verbunden. ${ }^{24}$

Dabei ist ebenfalls anzumerken, dass die weniger zeit-(und kosten-)intensiveren Kurse meist direkt von den Weiterbildungszentren der ortsansässigen Universitäten angeboten werden. Diese umfassen jeweils zumeist ein bis zwei Tage und nur wenige Stunden. Jedoch bedeutet das ebenfalls, dass diese Kurse häufig nur ein sehr geringes Feld an Teilbereichen der digitalisierungsbezogenen Kompetenzen abdecken. Das heisst, dass es dahingehend in der Verantwortung der Hochschuldozierenden selbst liegt, ihre digitalisierungsbezogenen Kompetenzen in der Lehramtsausbildung zu erweitern und ganzheitlich zu fördern bzw. ihre Defizite auszugleichen.

23 Unter den recherchierten Weiterbildungsangeboten befinden sich nur öffentlich beworbene Weiterbildungen. Interne Adhoc-Angebote seitens der Hochschulen, die als Reaktion auf die Pandemie kurzfristig für die in der Lehre tätigen Angestellten entworfen und angeboten worden sind, wurden hier nicht erfasst. Gerade in Anbetracht der Krisensituation waren neben Weiterbildungsangeboten auch andere Formen der Unterstützung für die Lehrenden von zentraler Bedeutung; beispielsweise «Information (zum Selbstlernen), Beratung, Kooperation (mit Experten) und Austausch mit Kollegen. Inhaltlich kann die Unterstützung rein technischer, didaktischer oder sozialer Art sein» (Reinmann, 2020, 13).

24 Preisintensive Angebote, wie «Digitalisierung in den Handlungsfeldern der Kinder- und Jugendarbeit» für 675,- Euro des IfW oder der Zertifikatskurs «Medienpädagogische Praxis» für 890,- Euro der Clearingstelle Medienkompetenz der Deutschen Bischofskonferenz an der KH Mainz bieten zwar ein breites Feld an adressierten Kompetenzbereichen, sind dafür aber sehr zeitintensiv mit einer Dauer von mehreren Monaten. Es gibt jedoch auch ausgedehnte Weiterbildungsangebote, deren Teilnahme geringe bis keine Kosten birgt: Die Angebote des JFF - Institut für Medienpädagogik in Forschung und Praxis unter dem Namen «Medien_Weiter_Bildung» erstrecken sich als Blended-Learning-Workshop über mehr als ein halbes Jahr und bleiben dennoch kostenfrei. 
In Bezug auf die Abdeckung der Teilbereiche des Kompetenzrahmens (siehe Tabelle 3) werden einige Defizite deutlich. In Abbildung 5 ist klar erkennbar, dass bei $n$ = 37 Weiterbildungsangeboten aus der Recherche die Eigene Medienkompetenz (26 Mal direkt adressiert) und die Mediendidaktische Kompetenz (30 Mal direkt adressiert) sehr stark repräsentiert werden. Auffällig ist auch, dass diese Teilbereiche vorrangig direkt adressiert werden und in wenigen Weiterbildungsveranstaltungen eine individuelle Transferleistung der Teilnehmenden notwendig ist, um den Kompetenzbereich zu fördern bzw. zu unterstützen.

Verteilung bei $n=37$

38

30

30

26

15

26

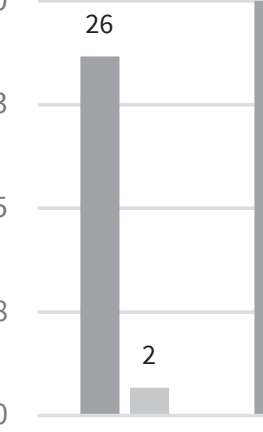

MK

MdK

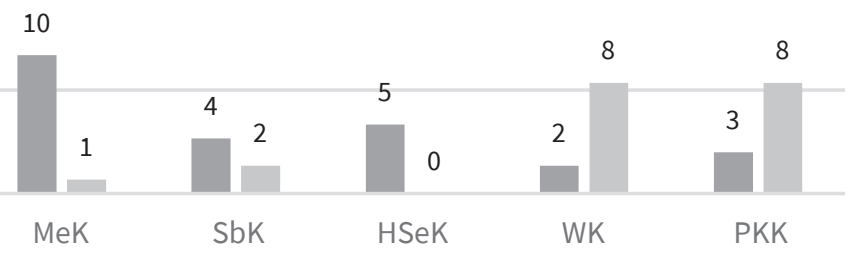

- Direkt 1 Indirekter Transfer

Abb. 2.: Verteilung der Kompetenzbereiche bei $n=37$ Weiterbildungsangeboten.

Jedoch sollten auch die anderen Teilbereiche des Kompetenzrahmens, wie die Medienerzieherische Kompetenz, die Sozialisationsbezogene Kompetenz im Medienzusammenhang, die Hochschulentwicklungskompetenz im Zusammenhang mit digitalen Medien sowie die Wissenschaftliche Kompetenz in Bezug auf digitale Medien und die Professionalisierung beruflicher Kommunikations- und Kollaborationskompetenzen, in Kursen und Workshops stärker vertreten sein, um diese zielbringend fördern zu können. In der Auswertung der Recherche wurde jedoch deutlich, dass die übrigen Bereiche seltener adressiert und gefördert werden: die Medienerzieherische Kompetenz ist mit zehn Zuordnungen (siehe Abbildung 2) vergleichsweise stark repräsentiert und wird dabei vorrangig direkt adressiert. Ebenfalls sind die Sozialisationsbezogene Kompetenz sowie die Hochschulentwicklungskompetenz mit drei bis fünf Zuordnungen bei direktem Adressieren dieser Bereiche zwar sehr gering vertreten, jedoch sind die Teilnehmenden weniger häufig auf individuelle Transferleistungen angewiesen. Die Wissenschaftliche Kompetenz und die Professionalisierung beruflicher Kommunikations- und Kollaborationskompetenzen können in den dargestellten 
Weiterbildungsangeboten fast ausschliesslich durch eigens initiierte Transferleistungen der Teilnehmenden gefördert werden. Dabei ist auf die Umfrageergebnisse zu verweisen, in denen deutlich wurde, dass besonders diese beiden Teilbereiche starke Wissenslücken aufweisen und Unterstützung bedürfen.

Zusammenfassend sind in den recherchierten Weiterbildungsangeboten besonders die Förderung der Eigenen Medienkompetenz sowie der Mediendidaktischen Kompetenz vertreten. Gleichzeitig sind Defizite in Bezug auf die Eigene Medienkompetenz im Rahmen der vorangestellten Umfrage per Selbsteinschätzung deutlich geworden. Anhand der Recherche lässt sich schlussfolgern, dass die in der Selbsteinschätzungsumfrage ausgemachten Wissenslücken in Bezug auf die Eigene Medienkompetenz mit den dargestellten Angeboten geschlossen werden könnten. Weitere Kompetenzbereiche, die bei den Hochschullehrenden als defizitär identifiziert wurden (Medienerzieherische Kompetenz, Wissenschaftliche Kompetenz und Professionalisierung beruflicher Kommunikations- und Kollaborationskompetenzen), sind in (teilweise sehr) geringer Zahl vertreten, obwohl Bedarf bestünde.

Aufgrund der CoViD-19-Pandemie liegt der Fokus aktuell geplanter Weiterbildungsangebote unumgänglich auf den Standpfeilern, die eine Online-Lehre begünstigen: die Eigene Medienkompetenz und die Mediendidaktische Kompetenz. Doch auch im Hinblick auf Angebote vor der Pandemie wird deutlich, dass die Pandemie zwar die erhöhte Frequenz dieser Schwerpunkte bedingt, jedoch nicht die allgemeine Vormacht vor den übrigen Aspekten digitalisierungsbezogener Kompetenz in Bezug auf die Lehramtsausbildung begründen darf.

\section{Handlungsempfehlungen zur Stärkung der Weiterbildungslandschaft}

Eine Gegenüberstellung der erhobenen Bedarfe im Monitor und der Kompetenzschwerpunkte in den Ausrichtungen der Weiterbildungsangebote für Hochschullehrende hat bereits erste Widersprüche gezeigt. Im Folgenden werden nun ausgehend von den Ergebnissen des Monitors sowie den Weiterbildungsangeboten Handlungsempfehlungen abgeleitet.

Basierend auf den in der Umfrage erhobenen Förderbedarfen lassen sich anhand der Recherche zu den Weiterbildungsangeboten ebenfalls Lücken aufzeigen, welche es zu schliessen gilt. Es bestehen Bedarfe hinsichtlich der Eigenen Medienkompetenz, der Medienerzieherischen Kompetenz, der Wissenschaftlichen Kompetenz in Bezug auf digitale Medien sowie der Professionalisierung beruflicher Kommunikations- und Kollaborationskompetenzen.

Anhand der Recherche wurde deutlich, dass die Eigene Medienkompetenz in den Weiterbildungen stark repräsentiert ist. Zu den vorrangig indirekt angesprochenen Kompetenzbereichen gehört die Professionalisierung beruflicher Kommunikations- und Kollaborationskompetenzen, die Wissenschaftliche Kompetenz in Bezug 
auf digitale Medien und die Hochschulentwicklungskompetenz - also oftmals direkt hochschulbezogene Kompetenzen, die die Medienpädagogische Kompetenz der Hochschullehrenden von der Medienpädagogischen Kompetenz der Lehrenden im Schulkontext unterscheidet. Hier besteht erhöhter Handlungsbedarf, da gerade Hochschullehrende des Lehramtsstudiums über eine konkret hochschulbezogene Medienpädagogische Kompetenz verfügen müssen.

Darüber hinaus sollten Angebote, welche digitalisierungsbezogene Kompetenzen adressieren, jährlich angeboten werden, so wie es bereits mit rechtlichen Grundlagen (OER, Datenschutz, Urheberrecht) gehandhabt wird (siehe Abbildung 2). Sie sollten ausserdem verstärkt auf den hochschulbezogenen Kontext fokussiert angeboten werden.

Ein weiterer Punkt, welcher sich aus den Recherchen ergibt, ist, dass Themen, wie Datenschutz und Urheberrecht, die basierend auf dem Monitor in der Lehrpraxis eher wenig präsent sind, in den dargestellten Weiter- und Fortbildungen stark im Fokus stehen. Eine stärkere Bewerbung solcher Angebote könnte diesem Defizit in der Lehrpraxis entgegenwirken.

Die Recherche ergab darüber hinaus, dass es bereits eine hohe Bandbreite an Weiterbildungen im digitalisierungsbezogenen Kontext gibt, diese jedoch nicht speziell auf Hochschuldozierende in Lehramtsstudiengängen ausgerichtet sind. Somit ergibt sich stets der Bedarf einer individuellen Transferleistung durch die Teilnehmenden in den Weiterbildungen, diese auf die eigene Lehre in der Lehramtsausbildung zu übertragen.

\section{Fazit}

Die Auswirkungen der CoViD-19-Pandemie auf die Qualität der Hochschullehre sind insgesamt noch als offen einzuschätzen (vgl. Crawford et al., 2020, 20). Basierend auf den Ergebnissen aus der Befragung der Hochschullehrenden in Lehramtsstudiengängen der TU Dresden verweist die durchgeführte Studie jedoch auf Lücken im Weiterbildungsangebot hinsichtlich der festgestellten Unterstützungsbedarfe. Die Befragung ergab, dass Bedarfe hinsichtlich der Eigenen Medienkompetenz, der Medienerzieherischen Kompetenz, der Wissenschaftlichen Kompetenz in Bezug auf digitale Medien sowie der Professionalisierung beruflicher Kommunikations- und Kollaborationskompetenzen bestehen. Auf die verschiedenen Kompetenzbereiche wird im Weiterbildungsangebot unterschiedlich stark eingegangen.

Anhand der Recherche zum Weiterbildungsangebot konnte aufgezeigt werden, dass die Eigene Medienkompetenz in den Weiterbildungen am häufigsten thematisiert wurde. Sie wurde in zahlreichen Fällen direkt adressiert. Zu den Kompetenzbereichen, welche zumeist indirekt vermittelt wurden, gehört die Professionalisierung beruflicher Kommunikations- und Kollaborationskompetenzen, die Wissenschaftliche 
Kompetenz in Bezug auf digitale Medien und die Hochschulentwicklungskompetenz also direkt hochschulbezogene Kompetenzaspekte. Hier zeigt die vorliegende Studie einen dringenden Handlungsbedarf auf, handelt es sich doch um für die Kompetenzvermittlung an Lehramtsstudierende essentielle Kompetenzbereiche.

Die Untersuchung der Weiterbildungsangebote hat gezeigt, dass in den Angeboten die mediendidaktische Ebene und die Eigene Medienkompetenz sehr häufig adressiert werden. Gleichzeitig wird deutlich, dass mit Blick auf die übrigen Kompetenzbereiche nicht ausreichend Angebote zur Verfügung stehen und stattdessen ein Überhang seitens mediendidaktischer Kompetenz und Eigener Medienkompetenz festzustellen ist.

Insgesamt bietet die Studie einen Einblick in den Wissens- und Erfahrungsstand der befragten Hochschullehrenden in Lehramtsstudiengängen der TU Dresden zu Beginn der Pandemie sowie zu deren Fort- und Weiterbildungsbedarfen und -möglichkeiten bezüglich digitalisierungsbezogener Kompetenzen im Sommersemester 2020. Sie liefert damit neben den herausgearbeiteten Handlungsempfehlungen ausserdem eine Grundlage für die weitere Forschung. Aufbauend auf den hier vorgestellten Ergebnissen aus der Gegenüberstellung von Unterstützungsbedarfen und Weiterbildungsangeboten könnten sowohl umfassende, universitätsübergreifende Studien als auch Untersuchungen zum Einsatz verschiedener Weiterbildungsformate folgen.

\section{Literatur}

Baacke, Dieter. 1997. Medienpädagogik. Tübingen: Niemeyer.

Blömeke, Sigrid. 2000. Medienpädagogische Kompetenz: theoretische und empirische Fundierung eines zentralen Elements der Lehrerausbildung. 1. Aufl. München: KoPäd-Verl.

Bräutigam, Sandra, und Florian Schindler. 2021. «Zukunft des agilen Lernens in der wissenschaftlichen Weiterbildung». In Agiles Lernen im Unternehmen, herausgegeben von Jörg Longmuß, Gabriele Korge, Agnes Bauer, und Benjamin Höhne, 137-43. Berlin, Heidelberg: Springer Berlin Heidelberg. https://doi.org/10.1007/978-3-662-62013-7_15.

Crawford, Joseph, Kerryn Butler-Henderson, Jürgen Rudolph, Bashar Malkawi, Matt Golwatz, Rob Burton, Paola A. Magni, und Sophia Lam. 2020. "COVID-19: 20 Countries' Higher Education Intra-Period Digital Pedagogy Responses». Journal of Applied Learning \& Teaching 3 (1): 10-28. https://doi.org/10.37074/jalt.2020.3.1.7.

Dallmann, Christine. 2017. Schule. In Grundbegriffe Medienpädagogik, herausgegeben von Bernd Schorb, Anja Hartung-Griemberg, und Christine Dallmann, 367-374. München: KoPäd-Verl. München.

Duchêne, Lucas, Philipp Sauerwald, und Friedrich Schulten. 2020. «Digitale Lehre der Fakultät Maschinenbau im Sommersemester 2020». Technische Universität Dortmund. https://doi. org/10.17877/DE290R-21678. 
European Commission. 2017. «Digitale Kompetenz Lehrender: Europäischer Rahmen für die Digitale Kompetenz von Lehrenden (DigCompEdu)». https://ec.europa.eu/jrc/sites/jrcsh/ files/digcompedu_leaflet_de-2018-09-21pdf.pdf.

Graf, Tobias P, Rüdiger Hohls, Claudia Prinz, und Matthias Pohlig. 2020. «Auswertung der Online-Umfrage ,Digitale Lehre‘ von H-Soz-Kult». Auswertung Online-Umfrage. Berlin: Humboldt-Universität. https://www.hsozkult.de/debate/id/diskussionen-5123.

Holdener, Anita, Silke Bellanger, und Serina Mohr. 2016. «,Digitale Kompetenz' als hochschulweiter Bezugsrahmen in einem Strategieentwicklungsprozess». In Digitale Medien: Zusammenarbeit in der Bildung, herausgegeben von Josef Wachtler, Martin Ebner, Ortrun Gröblinger, Michael Kopp, Erwin Bratengeyer, Hans-Peter Steinbacher, Christian FreislebenTeutscher, und Christine Kapper. Bd. 71. Medien in der Wissenschaft. Waxmann: Münster. https://doi.org/10.25656/01:15780.

Krammer, Georg, Barbara Pflanzl, und Marlies Matischek-Jauk. 2020. «Aspekte der Online-Lehre und deren Zusammenhang mit positivem Erleben und Motivation bei Lehramtsstudierenden: Mixed-Method Befunde zu Beginn von COVID-19». Zeitschrift für Bildungsforschung, Dezember. https://doi.org/10.1007/s35834-020-00283-2.

Kultusministerkonferenz - KMK. 2016. «Bildung in der digitalen Welt: Strategie der Kultusministerkonferenz vom 08.12.2016». Kultusministerkonferenz - KMK. https://www.kmk.org/ fileadmin/Dateien/pdf/PresseUndAktuelles/2018/Digitalstrategie_2017_mit_Weiterbildung.pdf.

Lund, Andreas, und Toril Aagaard. 2020. «Digitalization of teacher education: Are we prepared for epistemic change?» Nordic Journal of Comparative and International Education (NJCIE) 4 (3-4): 56-71. https://doi.org/10.7577/njcie.3751.

Marinoni, Giorgio, Hilligje Van't Land, und Trine Jensen. 2020. «The impact of Covid-19 on higher education around the world». AU Global Survey Report. International Association of Universities. https://www.iau-aiu.net/IMG/pdf/iau_covid19_and_he_survey_report_final_may_2020.pdf.

Matveeva, Svetlana Valentinovna, Natalia Sergeevna Akatova, Yuriy Ivanovich Shcherbakov, und Nadezhda Victorovna Filinova. 2020. «Digitalization of Higher Education and Professional Development of Educators: Technologies and New Opportunities». Revista Amazonia Investiga 9 (29): 77-86. https://doi.org/10.34069/AI/2020.29.05.10.

Mayring, Philipp. 2015. Qualitative Inhaltsanalyse: Grundlagen und Techniken. 12., Überarbeitete Auflage. Weinheim Basel: Beltz Verlag.

Rashid, Shazia, und Sunishtha Singh Yadav. 2020. «Impact of Covid-19 Pandemic on Higher Education and Research». Indian Journal of Human Development 14 (2): 340-43. https:// doi.org/10.1177/0973703020946700.

Reinmann, Gabi, Carla Bohndick, Eileen Lübcke, Alexa Brase, Marikje Kaufmann, und Nele Groß. 2020. «Emergency Remote Teaching im Sommersemester 2020». Bericht zur Begleitforschung - Lehrendenbefragung. Hamburg: Universität Hamburg. https://www.hul.unihamburg.de/dateien/begleitforschung-bericht-lehrendenbefragung-barrierefrei.pdf. 
Schaarschmidt, Nadine, Juliane Tolle, Christine Dallmann, und Verena Odrig. 2020. «Digitalisierungsbezogene Kompetenzen von Lehrenden in den Lehramtsstudiengängen. Entwicklung eines Kompetenzrahmens». In Gemeinschaften in Neuen Medien. Von hybriden Realitäten zu hybriden Gemeinschaften, herausgegeben von Thomas Köhler, Eric Schoop, und Nina Kahnwald, 377-184. Dresden: TUDpress. https://nbn-resolving.org/urn:nbn:de:bsz:14qucosa2-741399.

Schaper, Niclas. 2020. «Entwicklung Und Validierung Eines Modells Zur E-Lehrkompetenz». MedienPädagogik: Zeitschrift für Theorie Und Praxis Der Medienbildung 37 (Medienpädagogik als Schlüsseldisziplin):313-42. https://doi.org/10.21240/mpaed/37/2020.07.17.X.

SMK - Sächsisches Staatsministerium für Kultu2017. «Medienbildung und Digitalisierung in der Schule: Kompetenzrahmen ,Kompetenzen in der digitalen Welt' der Kultusministerkonferenz, Fassung SMK Konzeption ,Medienbildung und Digitalisierung in der Schule'». https://www.medienbildung.sachsen.de/download/Kompetenzrahmen_Medienbildung_ SMK_Uebersicht.pdf.

Toquero, Cathy Mae. 2020. «Challenges and Opportunities for Higher Education amid the COVID-19 Pandemic: The Philippine Context». Pedagogical Research 5 (4): em0063. https:// doi.org/10.29333/pr/7947.

Tulodziecki, Gerhard. 2017. Thesen zu einem Rahmenplan für ein Studium der Medienpädagogik. merz. medien + erziehung, 61 (3), 59-65.

Tulodziecki, Gerhard. 2012. «Medienpädagogische Kompetenz und Standards in der Lehrerbildung». In Jahrbuch Medienpädagogik 9, herausgegeben von Renate Schulz-Zander, Birgit Eickelmann, Heinz Moser, Horst Niesyto, und Petra Grell, 271-97. Wiesbaden: VS Verlag für Sozialwissenschaften. https://doi.org/10.1007/978-3-531-94219-3_13.

Ugur, Naciye Guliz. 2020. «Digitalization in Higher Education: A Qualitative Approach». International Journal of Technology in Education and Science 4: 18-25. https://doi.org/10.46328/ ijtes.v4i1.24. 